Musées, Patrimoine et Culture scientifiques et techniques

$164 \mid 2016$

mars-avril 2016

\title{
Les musées départementaux de la Haute-Saône et Wikipédia : de l'institution à la contribution
}

\section{Nicolas Poulain}

\section{OpenEdition \\ Journals}

Édition électronique

URL : http://journals.openedition.org/ocim/1642

DOI : 10.4000/ocim.1642

ISSN : 2108-646X

Éditeur

OCIM

Édition imprimée

Date de publication : 1 mars 2016

Pagination : 21-27

ISSN : 0994-1908

Référence électronique

Nicolas Poulain, « Les musées départementaux de la Haute-Saône et Wikipédia : de l'institution à la contribution », La Lettre de l'OCIM [En ligne], 164 | 2016, mis en ligne le 01 mars 2017, consulté le 14 novembre 2019. URL : http://journals.openedition.org/ocim/1642 ; DOI : 10.4000/ocim.1642

Ce document a été généré automatiquement le 14 novembre 2019.

Tous droits réservés 


\title{
Les musées départementaux de la Haute-Saône et Wikipédia : de l'institution à la contribution
}

\author{
Nicolas Poulain
}

Le château de Champlitte abrite le musée départemental d'Arts et Traditions populaires.

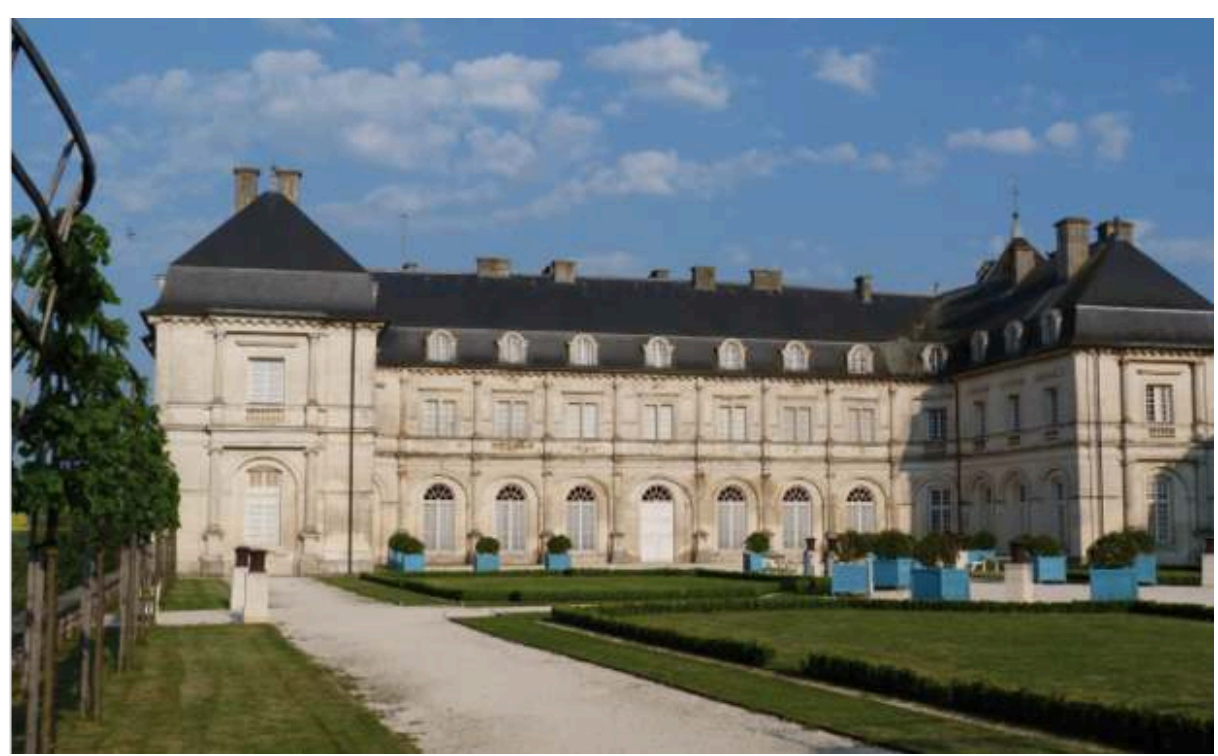

(C) Musée départemental d'Arts et Traditions populaires

\section{Le point de départ du projet}

1 Les musées départementaux de la Haute-Saône sont un réseau de 3 musées répartis entre Champlitte, à la frontière bourguignonne, et Château-Lambert à la frontière vosgienne. C'est une riche collection d'objets historiques, dits aussi ethnographiques, 
collectés dès 1952 par une famille dont l'initiateur, Albert Demard, est issu du monde rural. Cette collection occupe aujourd'hui l'ensemble d'un château néoclassique construit à la fin du XVIIIe siècle dans un bourg de presque 2000 habitants, Champlitte. Les deux autres musées du réseau ont été inaugurés plus tardivement, mais restent dans la lignée d'une muséologie propre à ces années, faits de reconstitutions, de maisons de plein air ou encore de patrimoine technique. Dans le droit fil du projet du musée national des Arts et Traditions Populaires (MNATP en son temps, actuel MUCEM), ses fondateurs ont prospecté leur territoire, "ont battu la campagne" à la recherche d'un "patrimoine en voie de disparition". Cet ancrage territorial reconnu débouche sur des pratiques de collecte extrêmement larges, variées, fournies... L'essentiel de ces collectes alimente un projet de reconstitution de l'ensemble des activités rurales dans le château pré-cité. Il s'agit donc, pour en référer à qui de droit mais à quelques arrangements près, d'unités écologiques où le monde est appréhendé, en dehors de quelques exceptions, par la diversité des activités humaines et professionnelles, artisanales et commerciales, que la famille Demard retient. Chaque unité est l'ombre de l'homme (ou des hommes) qui l'a produite et les salles se succèdent ainsi dans une approche à la fois fonctionnelle et sérielle : l'atelier du tisserand, les intérieurs paysans, le barbier, l'épicerie, la grange... en tout 40 salles pour le seul musée dit des Arts et Traditions populaires qu'une relecture "Hainardienne" nous ferait qualifier de muséographie d'auteur.

2 Je deviens chargé des collections de manière quasi synchrone avec le vote de la loi musée en 2002, l'instauration de l'obligation de récolement décennal qui en découle par décret en 2004, le lancement d'une politique active de numérisation du patrimoine coordonnée par la section fédérée de Franche-Comté de l'Association Générale des Conservateurs de Collections Publiques de France dans le cadre d'un contrat de plan état-région, et l'arrivée de Viviane Ivol à la direction des musées départementaux de la Haute-Saône.

3 Commence alors la mise en œuvre du plan de récolement qui verra en 12 ans l'informatisation de 20000 pièces (dont la documentation reste relativement pauvre, proportion élevée des collections en inventaire rétrospectif) et la numérisation de 12000 objets coordonnée par le musée mais assurée techniquement par des prestataires externes.

4 Nous décidons rapidement d'adosser les processus de récolement et de numérisation aux "unités écologiques" précédemment décrites, ce qui chaque fois demande une plongée dans un domaine qu'il soit technique (le travail du métal chez le forgeron, le travail du bois chez le sabotier, le travail de la terre...) ou thématique (les âges de la vie, la chasse...). À chaque thématique, l'article encyclopédique est une porte d'entrée, sorte de tour d'horizon synthétique ou de panorama sémantique dont on sait d'avance qu'il faudra le dépasser. Wikipédia joue ce rôle ; non que l'encyclopédie serve de référence, mais de première approche parfois fournie et pertinente, parfois décevante. Loin d'être une fin en soi, il s'agit de reconnaître la facilité d'accès et la qualité variable des contenus en ligne... une source parmi d'autres, c'est-à-dire un contenu à croiser, à vérifier, à corroborer. Il s'agit aussi et surtout, comme nous le verrons plus loin, d'assimiler le fait que Wikipédia est une porte d'entrée dans des sujets culturels pour une majorité d'internautes.

5 De page décevante, je me souviens avoir consulté la page sur les jougs au moment du récolement de ceux présents dans les salles du musée. Une page de Wikipédia vide, très 
peu de références bibliographiques, pas d'images... Dans un premier temps, ce n'est pas un problème en soi, puisque nous avons les ressources et les méthodes pour avancer sans cela. Dans un second temps, c'est une réelle frustration puisqu'à l'issue d'un certain nombre de recherches et après numérisations, nous pourrions être en mesure de rédiger et d'illustrer la page sur le joug de Wikipédia, juste promotion des collections dans ce qu'elles apportent au savoir... Sauf qu'institutionnellement, c'est impossible pour des raisons que le cheminement de ce projet a en partie révélées, les préjugés et la frilosité sur la question de la réutilisation commerciale de reproductions de collections relevant du domaine public étant quelques-uns des principaux freins. Notre projet Wikipédia est né de ce constat d'impossibilité.

\section{Exemple d'affichage des métadonnées issues de l'inventaire transférées sur le site} commons.wikimedia.org

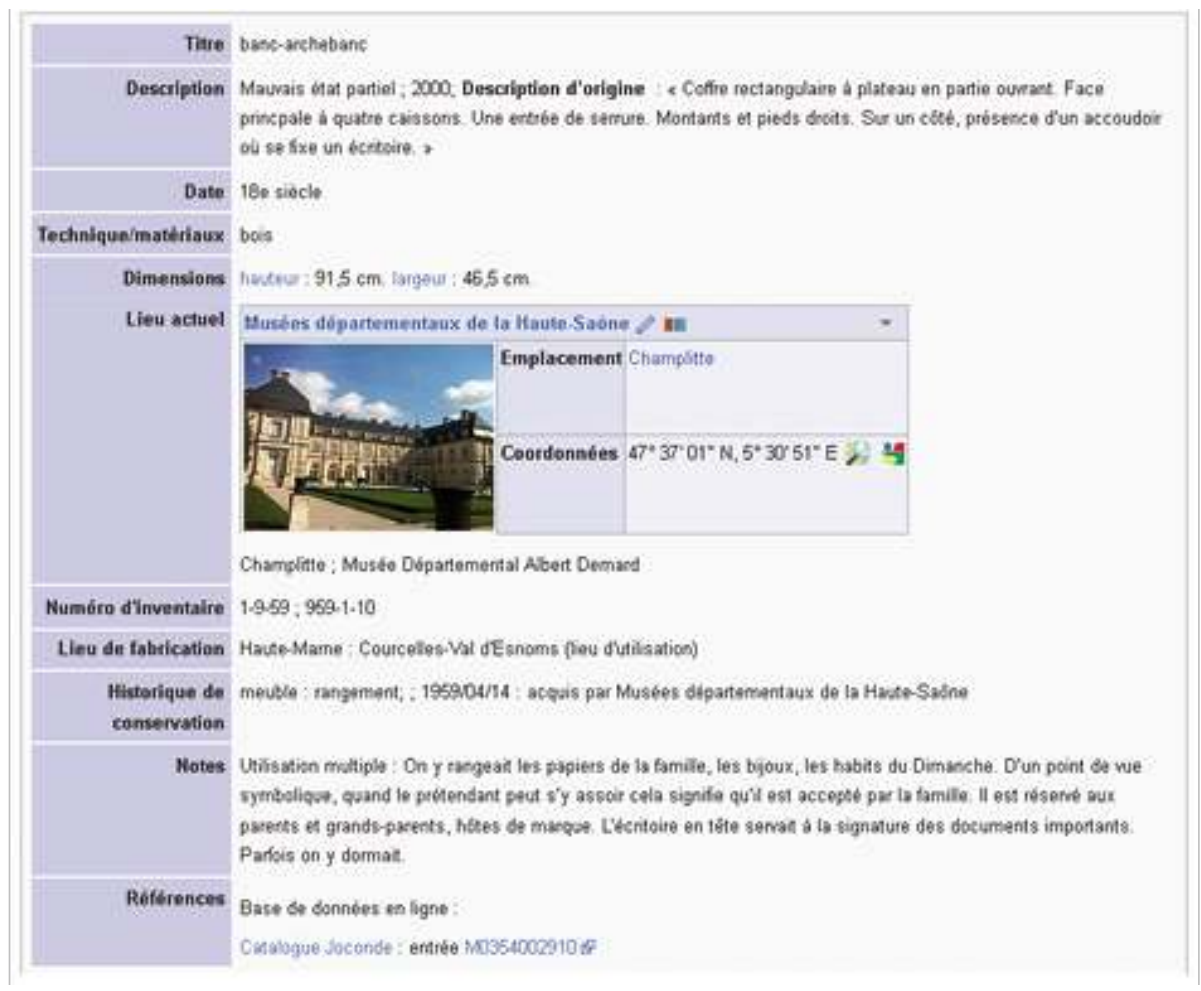

\section{L'écosystème Wikimédia}

6 À partir du moment où la participation d'une institution à Wikipédia est envisagée, se pose inévitablement la complexe question : "Wikipédia c'est quoi ?".

7 En soi et pour commencer, un musée n'a pas besoin d'autorisations, de conventions ou même de compte pour écrire sur Wikipédia puisque c'est le principe même: une encyclopédie collaborative en ligne et donc écrite à plusieurs mains, quelles qu'elles soient.

8 Quelques chiffres : 1200000 articles en français, une majorité d'articles sur la culture et les arts, est considérée par plus de la moitié des Français comme une source d'information pour le travail ou la culture générale, 6e site en France, 1er site culturel, 19 millions de visiteurs par mois. Wikipédia c'est surtout une douzaine de projets, et 
pour n'en citer que quelques-uns: Wikimedia Commonsune (une base de données multimédia, illustrant entre autre l'encyclopédie Wikipédia), Wiktionnaire (un dictionnaire), Wikiquote (un recueil de citations) ou encore Wikibooks (une base de livres et textes didactiques). L'ensemble de ces projets est réalisé sous l'égide de la Wikimedia Foundation, fondation américaine basée en Californie mais qui n'a pas de regard direct sur le contenu de chacun de ces sites.

En effet, le point commun de ces projets est qu'ils sont collaboratifs pour la création et la modification de leur contenu et sont basés sur sa libre réutilisation. La libre réutilisation signifie notamment que les images et les textes présents sur Wikipédia sont exploitables commercialement par tous ceux qui s'engagent à respecter la redistributivité du contenu. Autrement dit, on peut exploiter, commercialement ou pas, le contenu présent sur Wikipédia si le contenu ainsi créé avec est lui-même réexploitable selon ces mêmes conditions. C'est la révolution des licences libres, plus particulièrement des licences Creative Commons créées dans les années 2000 et maintenant validées juridiquement en France ${ }^{1}$.

Vous avez dit Libre?

Chaque fois qu'il est mention de donnée(s) libre(s), ce terme fait référence à l'expression "logiciel libre" né dans les années 1980, formalisée pour l'informatique par Richard Stallman. Par la suite étendue à la notion de production intellectuelle (donc relevant du domaine de la propriété intellectuelle), cette expression fait référence à 4 libertés fondamentales qui sont les suivantes : la possibilité d'utiliser l'œuvre, pour tous les usages ; la possibilité d'étudier l'œuvre ; la possibilité de redistribuer des copies de l'œuvre ; la possibilité de modifier l'œuvre et de publier ses modifications.

Ces droits s'entendent y compris pour des utilisations commerciales. Il ne faut pas confondre libre et gratuit, de la même manière qu'il faut distinguer la diffusion (notion d'accès) de la réutilisation (notion d'exploitation).

Aujourd'hui de nombreuses licences sont dites compatibles avec cette définition. C'est le cas de certaines licences Creative Commons (pas toutes) ou de la licence ETALAB (publiée en 2011, www.etalab.gouv.fr/licence-ouverte-open-licence) récemment créée par l'État français.

10 On voit donc que si Wikipédia n'appartient pas à la Wikimedia Foundation, cette encyclopédie appartient en fait à tous. La fondation américaine s'occupe d'assurer l'infrastructure technique qui porte les projets Wikimedia (de manière très concrète, les serveurs qui hébergent les projets et les logiciels qui permettent leur mise en ligne).

11 Au-delà de notre envie de "juste retour", il s'agissait aussi de construire un projet cohérent autour de notre participation à Wikipédia. C'est à ce point que rentrent en jeu les chapitres. Ce sont des organisations indépendantes créées pour soutenir et promouvoir les projets Wikimédia dans une région géographique donnée (un pays par exemple). Ces chapitres visent à favoriser le développement de contenu sous licence libre autour ou pour les projets Wikimedia. En France, il s'agit de Wikimedia France, sous statut associatif, qui œuvre depuis plusieurs années auprès des institutions patrimoniales, notamment auprès des collections publiques françaises pour en favoriser la diffusion en France et dans le monde. En 2012, Rémi Mathis, conservateur à 
la Bibliothèque nationale de France, alors président de Wikimedia France et Adrienne Charmet-Alix, responsable des partenariats GLAM, sont des interlocuteurs privilégiés pour les projets liés au patrimoine. Certains partenariats ont déjà été menés entre Wikimedia France et des institutions patrimoniales : la ville de Toulouse, le château de Versailles, le musée de Cluny... partenariats qui ont fait l'objet de conventionnements sur lesquels nous nous sommes appuyés pour construire le nôtre.

Enfin et surtout, l'écosystème Wikimédia, ce sont les wikipédiens, communauté d'utilisateurs rompus au fonctionnement de l'encyclopédie. Ces bénévoles, membres ou pas de Wikimédia France, sont des chevilles ouvrières, et ce, à différents niveaux d'intervention: que ce soit techniquement en créant l'environnement de travail de l'institution, soit pragmatiquement en étant garant du respect des principes de coproduction des contenus ou servant de guide dans l'utilisation des différents outils de collaboration (forums, page projet, réalisation des modèles...).

\section{Exemple de mentions y compris des droits liées à une image (capture d'écran du site} commons.wikimedia.org)

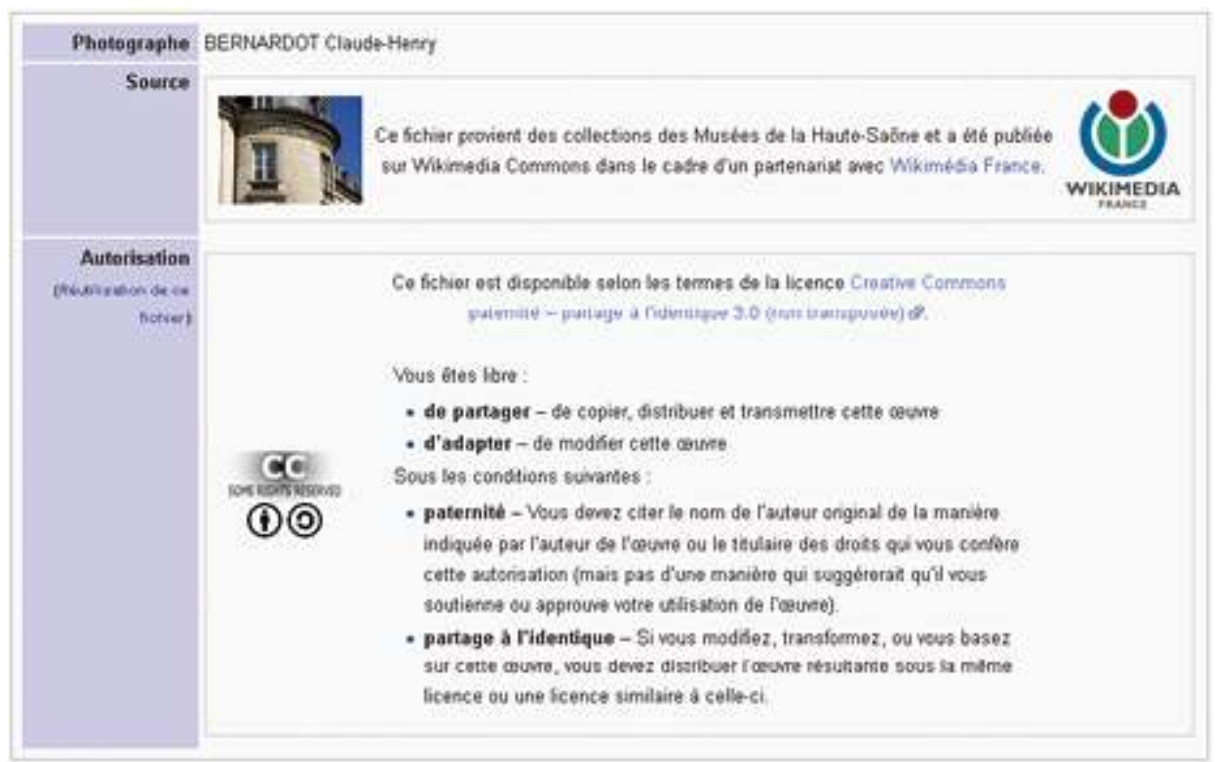

\section{Les contraintes techniques et les solutions trouvées}

L'objet du partenariat avec Wikipedia vise l'amélioration de l'encyclopédie, et c'est cet objectif qui nous réunit. Mais l'intérêt de notre institution va tout de suite au-delà. Il s'agit pour nous de donner de la visibilité à la collection des musées départementaux, et à travers elle à la collectivité détentrice de cette collection; d'envisager l'enrichissement de cette collection et des données associées via cette mise en ligne ; il s'agit surtout de diffuser de la culture, mission première de nos institutions. Le partenariat s'est vite orienté vers un versement massif de données sur WikimediaCommons, la base multimédia, données à valoriser dans les articles de Wikipédia. Wikimedia France s'engage à nous fournir l'assistance technique nécessaire à la mise en œuvre ou encore à fournir des statistiques sur l'utilisation de nos données. Cette convention est signée en décembre 2012 après les différentes validations des services de notre collectivité. 

fonctionnement administratif est peu habitué à traiter.

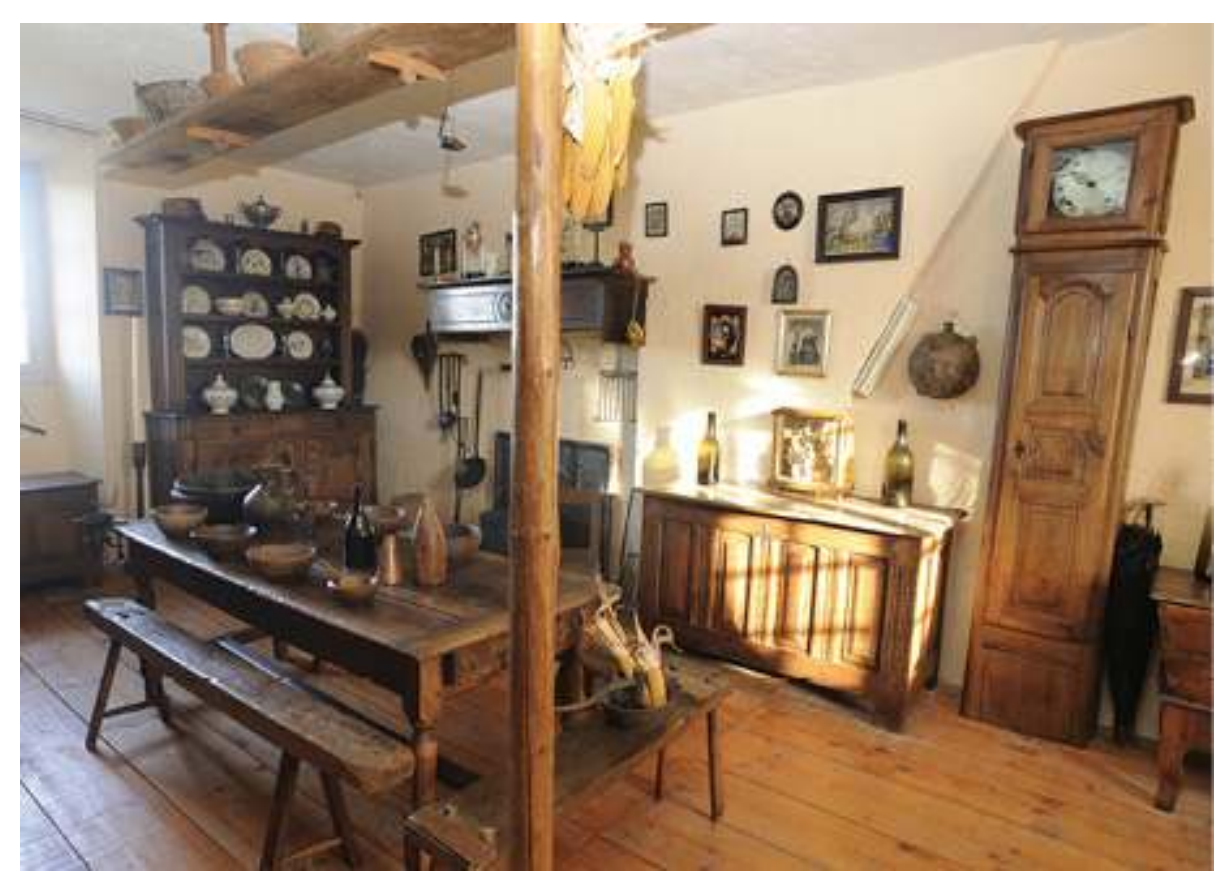

(ㄷ) Musée départemental d'Arts et Traditions populaires

En complément du musée, les services concernés sont les services juridiques, informatiques et communication au sein d'une même collectivité. Service juridique, car la majorité des cessions de droits, quand elles existent, ont rarement pris en compte l'existence des licences libres. Service informatique, qui est un relais essentiel dans la manipulation des données, l'exploitation du système de gestion de données ou dans la définition des autorisations techniques qui rendent le dialogue possible entre les systèmes informatiques. Service communication dans la mesure où la diffusion sur Internet mobilise l'image de la collectivité. Dans un contexte ancré de maîtrise et de contrôle de l'image, il est par exemple difficile mais incontournable de questionner les circuits de validation classiques qui sont hiérarchiques, verticaux et longs; ces questions sont analogues à celles soulevées dans la mise en œuvre de comptes officiels d'institution sur les réseaux sociaux numériques.

16 Même identifiés, ces partenaires indispensables sont eux aussi en face de problématiques qu'ils n'ont pas l'habitude de traiter : travailler avec des licences libres pour un service juridique, laisser un accès à 10000 images pour un service informatique... sont des demandes extraordinaires, rappelant par là même cette dimension essentielle de l'expérimentation (culturelle) des pratiques muséales qu'elles soient numériques ou pas.

17 Les objectifs redéfinis du projet une fois construit deviennent alors : la diffusion de la connaissance via les données culturelles produites par le service, la valorisation du département de la Haute-Saône, la maîtrise de (ou participation à) l'image de l'institution sur Internet, l'amélioration de nos connaissances et enfin la participation à un projet collectif et ambitieux. Et ces objectifs rencontrent l'adhésion tant au niveau 
des interlocuteurs politiques que des services administratifs évoqués. Les changements de pratiques que cela implique dans le futur nourrissent le débat sur l'harmonisation de ses pratiques entre les services culturels.

Il reste toutefois beaucoup plus difficile de faire valoir les enjeux liés à l'arrivée du web de données ou le rôle prépondérant que joue Wikipédia dans la définition des modèles du web sémantique. Le web sémantique, vu comme composante du web 3.0 consiste à décrire des ressources disponibles sur Internet de manière à faciliter l'automatisation de leur traitement. Les infobox musées ou œuvre d'art sur Wikipédia sont une manière de décrire ou de sémantiser les ressources liées à un musée ou une œuvre d'art. Cette normalisation permet à des systèmes informatiques de comprendre de manière détaillée ces informations. C'est le cœur du projet SemanticPedia lancé en 2012.

\section{Un ensemble d'actions}

19 Le socle du projet était donc la possibilité de contribuer : le cadre juridique était le préliminaire à toute autre action. Deux points étaient à articuler dans ce domaine : la possibilité de basculer les images produites par le musée comme support de contribution sur l'encyclopédie Wikipédia et la possibilité pour les agents du musée d'avoir une information claire sur leur possibilité de contribuer.

Nous avons pu programmer des séances d'initiation à la contribution au sein de l'équipe, envisagée comme premier promoteur du projet. Ces ateliers ont ouvert entre nous des débats très intéressants sur la notion de source et de statut de l'information... Les questions de confiance et d'esprit critique ont tout de suite émergé pour réfléchir collectivement sur la teneur des informations qu'une institution diffuse. Dans ce cas, la manière dont l'écosystème Wikipédia fonctionne permet de mettre le doigt sur le statut de l'information et de relativiser toute information en soi en regard des sources qu'elle mobilise. Dans notre contexte de documentation éparse concernant les collections, le gain est double : il permet de distancier une partie de la documentation orale liée aux collections du musée, il fonde également les bonnes pratiques de rédaction sur Wikipédia dans le sens où les contributions sont envisagées d'emblée avec leurs sources. Le deuxième atelier a été réalisé la semaine de la MuseumWeek, semaine de valorisation des musées sur Twitter, et a donc été l'objet d'une mise en avant via ce canal de diffusion.

21 Le déplacement du projet vers l'action culturelle est essentiel. Cela permet de donner sens aux dispositifs juridiques mis en place, d'aller vers le double avantage de visibilité de la structure, d'enrichissement de l'encyclopédie et cela permet aussi de tendre vers l'autonomisation du projet, on pourrait dire "amorcer la pompe du dispositif participatif".

22 Trois autres actions, avec des retours plus ou moins conséquents, ont donc nourri ce partenariat :

23 - D'abord, nous avons adossé un concours photo ayant pour sujet le château de Champlitte, bâtiment classé hébergeant le musée, au concours organisé par Wikimédia France. Ce concours appelé "Wiki Loves Monuments" a lieu chaque année ; il a pour but de favoriser l'illustration de tous les bâtiments classés sur Wikimedia Commons. Là encore il s'agissait d'impliquer notre public dans la production d'images sous licence libre. Les retombées sont multiples, et au-delà de l'émulation, cela crée une banque 
d'images très aisée à utiliser pour les personnes en recherche d'iconographie sur le château-musée ;

- Ensuite, nous avons mis en œuvre la contribution systématique par trois des agents du musée sur les pages qui concernent directement les musées départementaux. L'objectif était l'amélioration des données reprises par les moteurs de recherche; en effet, dans de nombreux dispositifs de recherche (très populaires comme Google, Facebook ou qui préfigurent le web de données comme l'expérience d'annuaire de musées http:// labs.antidot.net/museesdefrance/) il s'agit des données libres issues de Wikipédia qui constituent le premier niveau d'information accessible. Ce systématisme a l'autre avantage de valoriser toutes les productions du musée comme source sur des sujets variés, revenant ainsi sur notre mission de création/diffusion de nos productions scientifiques. Pour les musées départementaux, nous avons ainsi valorisé nos catalogues d'exposition, nos productions sonores et nos expositions itinérantes. Par exemple, l'article "Château de Champlitte" reprend l'ensemble de l'iconographie historique présente dans les collections du musée, s'appuie abondamment sur le catalogue de l'exposition La vie de château : de la forteresse à la résidence de plaisance (2011) et des recherches menées à cette occasion. Il fait référence également aux créations sonores produites par le musée et accessibles en ligne. Autre exemple, l'article sur le chanvre cite l'exposition itinérante produite par le musée, citation qui a été une porte d'entrée pour une partie de son exploitation (certaines demandes d'emprunt de l'exposition, notamment hors du réseau muséal, sont passées par ce référencement sur Wikipedia. Nous sommes là proche d'une frontière ténue entre la valorisation et la communication. La communauté des contributeurs reste garante de cette distinction que les acteurs du projet doivent questionner et débattre collectivement pour ne pas franchir les limites imposées par l'encyclopédie ;

- Enfin, nous avons programmé et piloté une journée de rencontres professionnelles autour de ces questions via la section fédérée de l'AGCCPF de Franche-Comté et accueillie par la DRAC Franche-Comté. Cette journée avait pour but d'introduire les professionnels à l'écosystème Wikimedia et d'évoquer un certain nombre d'expériences déjà menées autour du projet Wikipédia (voir les interventions de cette journée sur http://musees.haute-saone.fr/journee-professionnelle-wikipedia-et-musees). La sollicitation de l'OCIM pour cette journée a débouché sur la création d'une bibliographie commentée intitulée "Wikipédia et musées" et publiée sur le site de l'OCIM (www.ocim.fr/bibliographie/wikipedia-musees/). Cette bibliographie nourrie des références fournies par les intervenants comporte beaucoup de ressources en ligne. 
L'espace consacré au travail du chanvre : la photographie prise par un agent du musée illustre la page Wikipédia consacrée au musée.

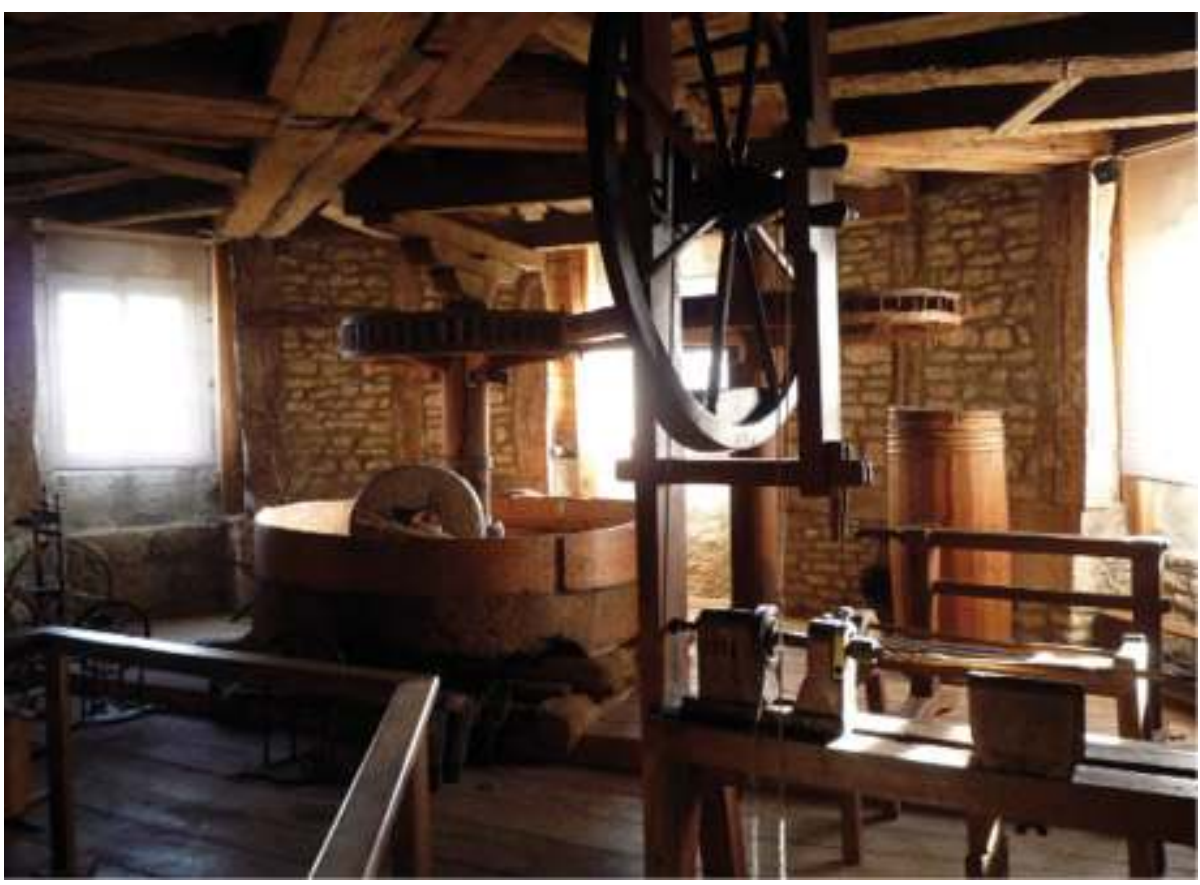

(c) Jérôme Bardiau, CC-BY-SA

\section{Data-manager, une nouvelle composante métier}

Dans la construction de ce type de projet, un des points cruciaux est d'être capable d'appréhender l'ensemble de la chaîne technique qui relève de ce que l'on peut nommer le data-management. Il s'agit d'une part, de maîtriser l'ensemble des enjeux et solutions juridiques (licences libres, diffusion web, open data) et d'autre part, de maitriser le cheminement des données d'un système vers un autre, en l'occurrence ici d'un outil de gestion des données normalisé vers un système ouvert et documenté, celui de Wikipédia.

De manière pragmatique, il faut réaliser une correspondance des données entre le modèle informatiquement quelque peu archaïque du Service des Musées de France ${ }^{2}$ avec des outils en train de se faire (en l'occurrence ici un outil de versement automatique appelé GLAM Wiki Toolset) dont l'utilisation reste complexe. Ces outils, relevant de prototypes fonctionnels, développés et maintenus par des wikimédiens bénévoles, demandent la participation à des discussions techniques entre une poignée de contributeurs européens, dont certains issus d'Europeana. 


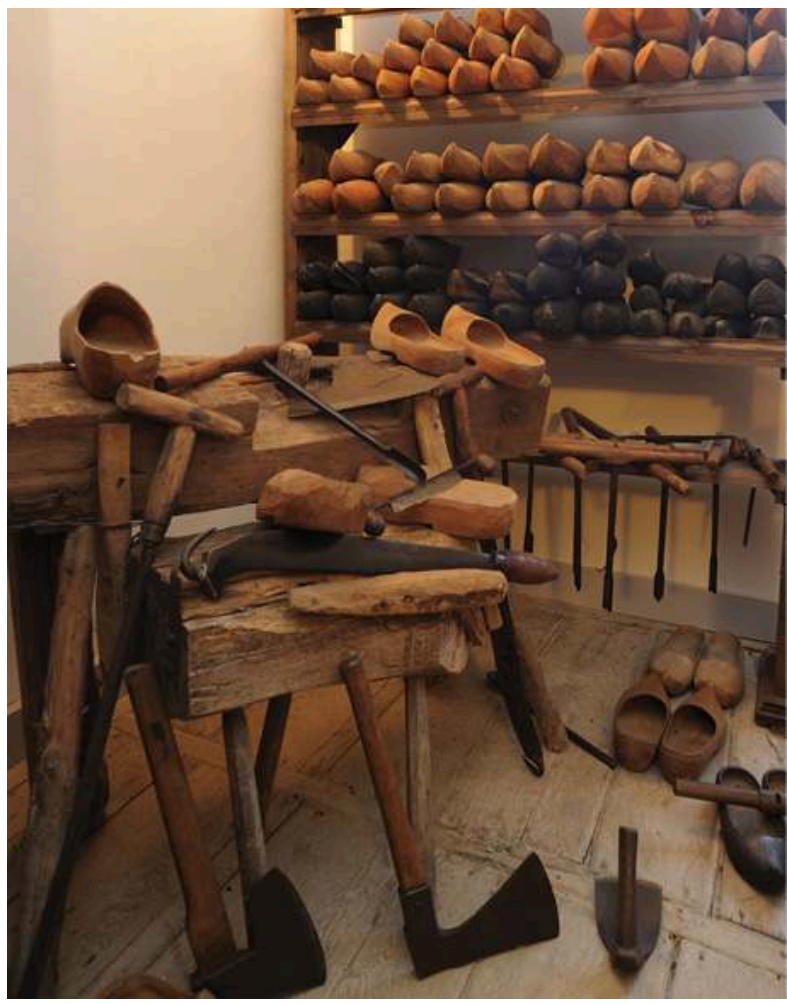

() Musée départemental d'Arts et Traditions populaires

Le travail sur les correspondances de données renvoie également les musées à leurs pratiques de normalisation et tout antécédent d'écart méthodologique est complexe à gérer. Nous restons à ce jour sur un échec technique en regard des choix opérés quant à la diffusion des images en haute définition initialement prévue, le choix de verser des fichiers haute définition au format TIF non compressé (environ $70 \mathrm{Mo}$ ) n'étant pas supporté par l'outil d'import automatique. Les retours qu'implique la démarche participative ainsi que l'ouverture de données posent également des questions importantes post-diffusion : comment gérer massivement des apports de connaissance via un système contributif ? De quel écosystème relèvent ces données ?... Enfin, ce type de dispositif révèle des débats très actuels d'usage et d'appropriation démocratique de la culture (droits liés aux numérisations des collections publiques, statut du domaine public, notion de Biens communs informationnels...) ${ }^{3}$, débats dans lesquels les voix officielles restent polyphoniques, entre ouverture de données ${ }^{4}$ et exception culturelle ${ }^{5}$ ). Dans les petites structures, cette compétence n'est, la plupart du temps, ni clairement identifiée, ni développée. Dans les grandes institutions, l'attribution du rôle fluctue au gré des projets et/ou des personnels.

29 Ces thématiques sont la source d'échanges professionnels notamment dans un groupe Facebook intitulé "Musées et collections : de la conservation au data-management".

\section{Bilan et perspectives}

Aujourd'hui, le cœur du projet consiste à avoir versé 2500 images issues des collections sur la banque d'images libres Wikimedia Commons, emboîtant ainsi le pas à de grandes 
institutions, du musée Guggenheim à New-York au musée des Augustins ou le muséum d'Histoire naturelle à Toulouse en passant par le Rijksmuseum d'Amsterdam. Sur ces 2500 images, la dimension participative a permis de voir une grande partie des métadonnées traduites en anglais améliorant ainsi leur accessibilité sur Internet, et la majorité des images catégorisées pour en faciliter l'accès également. Un faible pourcentage de cet ensemble sert d'illustration ou de source à des articles (170 utilisations au moment de l'écriture de cet article). Ce qui pose la nécessité indéniable de l'action culturelle au-delà de l'ouverture des données.

31 Ce domaine d'action reste actuellement très mouvant. Les implications évoluent au rythme des expériences menées par des institutions pionnières ; ce qui leur vaut, dans un premier temps au moins, une large reconnaissance sur les médias numériques et un relais très positif au niveau des promoteurs d'une culture accessible à tous mais force à la modestie... Il faut reconnaître enfin que ces projets sont rarement considérés comme structurants dans les institutions quelles qu'en soient leur taille. Et si l'on voit l'avantage indéniable de créer des collectifs hybrides (wikipédiens, public, amateurs, professionnels de musée), leur fragilité reste intrinsèque à cette forme de marginalité.

Pour conclure, une des pistes de réflexion pour l'avenir était de proposer un atelier numérique, rendez-vous régulier de contributions, d'échanges, d'expérimentation autour des connaissances liées au musée, sur le modèle des Fab Lab en milieu rural ou sur le modèle d'un Museomix permanent et ancré localement ${ }^{6}$. Un lieu de rencontre qui contribuerait peut-être à définir une des modalités relationnelles d'un musée avec son territoire, ou qui dessinerait la manière dont une population peut influer sur les dispositifs de médiation, de valorisation ou de réécriture muséographique (notamment numériques) à travers une démarche contributive et l'appropriation des artefacts patrimoniaux: en quelque sorte une mise au numérique de ce que sont les grandes lignes de l'écomuséologie.

L'auteur tient à remercier pour leur relecture et leur contribution: Viviane Ivol (directrice des Services Culturels, département de la Haute-Saône), Rémi Mathis, JeanFrédéric Berthelot et Anne-Laure Prévost (Wikimedia France), Christelle Molinié, Benoît Deshays (musée des Augustins de Toulouse) et l'équipe des musées départementaux de Haute-Saône.

\section{Vers un Ethnopôle sur les relations entre patrimoine et contribution} citoyenne

Les musées départementaux sont engagés dans la préfiguration d'un Ethnopôle (http://musees.haute-saone.fr/lethnopole), c'est-à-dire un label attribué par le ministère de la Culture-Direction des Patrimoines à une structure, qui articule recherche en sciences sociales et action culturelle en lien avec des musées.

Les démarches de préfiguration interrogent comment réinventer les musées d'Arts et Traditions Populaires en faisant des musées des lieux de "contributions citoyennes". Par l'enquête en ethnologie du présent, il s'agit d'aménager les conditions permettant aux citoyens d'apporter une part constructive à la définition de l'avenir des musées et de projets musées-territoires. À côté (ou à la suite) d'une contribution numérique, chacun est invité à prendre position sur la définition du futur Programme Scientifique et Culturel Contributif, à être acteur du sens des musées en animant des visites ("Suivez le guide Soyez le guide : venez 
raconter vos musées") ou en revisitant l'exposition permanente (Un musée habité, Gang des chiffonniers...).

Les collections du musée des Augustins de Toulouse : de l'open data au web sémantique via Wikidata

Le musée des Augustins de Toulouse a mené un projet de partenariat avec Wikimédia France (2011-2014) concomitant avec celui des musées de la HauteSaône. Bien que revêtant une forme sensiblement différente les préoccupations furent les mêmes : valoriser les collections, les ouvrir pour inciter le public à l'appropriation et à la réutilisation et expérimenter de nouveaux usages et outils. Les images des collections du musée des Augustins et leurs métadonnées ont été versées sur Wikimedia Commons. D'autre part des enseignants et leurs étudiants ont été invités à publier dans Wikipédia des articles relatifs au musée et à ses œuvres dans le cadre d'ateliers de contribution. Lorsque le musée a publié ses collections en open data sur la plateforme municipale dédiée, des bénévoles se sont spontanément emparés de ce jeu de données pour le croiser avec les images à disposition sur Wikimedia Commons et il a été possible de réaliser une des premières créations massives d'éléments d'œuvres d'art dans la base de données Wikidata.

L'objectif était de publier les métadonnées d'inventaire des collections sous la forme de données structurées Wikidata afin de créer des liens sémantiques entre les différents projets Wikimedia (Wikimedia Commons, Wikipédia) et les bases de données et référentiels en ligne préexistants (base de données du site du musée, Joconde...) et de les rendre interopérables. Des extractions de la base d'inventaire et un travail d'harmonisation et d'alignement des données ont permis aux bénévoles de procéder au versement dans Wikidata d'une partie conséquente des collections du musée figurant dans le domaine public. Ces données sont placées sous licence $\mathrm{CC} 0$, c'est à dire sans aucune restriction de droit. Ce jeu de données constitue un référentiel et une matière première pour des réutilisations ultérieures et a déjà alimenté des projets innovants comme le mashup "Les Musées en France" (site Internet qui croise différentes sources ouvertes pour constitué un annuaire enrichi et autonome des musées labélisés) et le moteur de recherche multilingue "Crotos" démontrant l'intérêt pour les institutions qui voient en retour leurs contenus enrichis.

Christelle Molinié et Benoît Deshays

Musée des Augustins à Toulouse 


\section{NOTES}

1. Adaptées en France à partir de 2003 dans le cadre du Centre d'Études et de Recherches de Sciences Administratives et Politiques/CNRS, notamment par Mélanie Dulong de Rosnay.

2. Ce schéma d'échanges de données a sa raison d'être historiquement comme s'en explique le Service des Musées de France via Laurent Manœuvre. Pour plus d'informations, voir l'espace professionnel sur le site Joconde www.culture.gouv.fr/documentation/joconde/fr/partenaires/ AIDEMUSEES/aide-musees.htm

3. Voir à ce propos le site et les réflexions de Lionel Maurel, juriste et bibliothécaire http:// scinfolex.com/ et le site du collectif Savoirs communs www.savoirscom1.info ; voir également les rencontres professionnelles Les musées de France face aux nouveaux enjeux du numérique qui ont eu lieu en septembre 2015 organisées par le Service des Musées de France.

4. Lire à ce titre le rapport Data Culture de Camille Domange par exemple ou encore le discours inaugural de SémanticPédia d'Aurélie Filipetti le 19/11/2012 : "nos établissements sont invités à leur tour à consolider, à enrichir ou à rectifier s'il y a lieu les données figurant sur Wikipédia, afin non seulement d'augmenter la qualité de ses articles en langue française, mais aussi son efficacité sur le Web sémantique." www.culturecommunication.gouv.fr/Presse/Discours/Lancement-DBpedia-enfrancais-et-inauguration-de-Semanticpedia.

5. Le fameux article 11 de la loi de 1978 sur l'utilisation des données publiques qui institue la dérogation concernant les données culturelles et permet aux établissements de fixer eux-mêmes les conditions de réutilisation des données qu'ils produisent.,

6. Museomix est une rencontre de médiateurs, bricoleurs, designers, développeurs, graphistes, communicants, artistes, écrivains, scientifiques... qui se retrouvent au cœur d'un musée pour un marathon créatif, intensif, festif de 3 jours (www.museomix.org).

\section{RÉSUMÉS}

À travers cette présentation de la participation d'un réseau de musées à l'encyclopédie Wikipédia, l'auteur décrit les principales étapes du projet, les problématiques (juridiques, informatique, communication) mises en jeu par la diffusion de donnés sous licence libre, les objectifs visés (visibilité des collections et de la collectivité territoriale) montrant l'importance de ce type d'expérimentation culturelle dans les pratiques du musée.

\section{INDEX}

Mots-clés : musée départementaux de la Haute-Saône, wikipedia, licence libre 


\section{AUTEUR}

\section{NICOLAS POULAIN}

assistant de conservation, chargé des collections au musée d'Art et d'Histoire de Saint-Brieuc nicolas.poulain@ouvaton.org 